\title{
Syzygies on Path Algebras
}

\author{
Sylvain Attan ${ }^{1}$, Andre Saint Eudes Mialebama Bouesso ${ }^{2 *}$ \\ 1 Université D’Abomey-Calavi, Département De Mathématiques, 01 Bp 4521, Cotonou 01, Bénin. \\ 2 Stellenbosch University, African Institute For Mathematical Sciences, 6 Melrose Road, Muizenberg, Cape \\ Town 7945, South Africa.
}

* Corresponding author. Tel.: +27631105151; email: MIALEBAMA@AIMS.AC.ZA

Manuscript submitted April 10, 2017; accepted September 11, 2017.

doi: 10.17706/ijapm.2017.7.4.224-240

\begin{abstract}
Let $K$ be a field and $K Q$ be a noetherian path algebra for the quiver $Q$. Given a left (resp. right) finitely generated ideal $I$ of $K Q$, we propose a new idea for computing left (resp. right) Groebner bases on $K Q$. As application, we propose a method for computing the so called left (resp. right) syzygies, that is, given polynomials $f_{1}, \ldots, f_{s} \in K Q \backslash\{0\}$ we propose a method for computing the set of all elements $\left(h_{1}, \ldots, h_{s}\right) \in(K Q)^{s}$ such that $h_{1} f_{1}+\ldots+h_{s} f_{s}=0$ (resp. $f_{1} h_{1}+\ldots+f_{s} h_{s}=0$ ).
\end{abstract}

Key words: Groebner bases, path algebra, syzygies.

\section{Introduction}

The advent of Groebner bases made a lot of computation possible in different areas of mathematics especially in algebraic geometry and commutative algebra. The theory of Groebner bases is widely studied in the commutative as well as non-commutative case over free associative algebras (see [1]-[7]) with their applications in computing syzygies [8]-[10]. The goal of this work is to revisit the non-commutative Groebner bases over path algebras. We propose a new approach for computing left and right Groebner bases on path algebras. Beside the left and right S-polynomials, we introduce the notion of left and right extended S-polynomials which is suitable for path algebras. Our approach is a generalization of the idea used in [1] and [9] chapter 2 which enables to compute a left and right Groebner basis using only selected $S$-polynomials in the Buchberger's criterion. We generalize the Schreyer's theorem on path algebras and as application we propose a method for computing left and right syzygies modules on path algebras i.e given polynomails $f_{1}, \ldots, f_{s} \in K Q \backslash\{0\}$ we propose a method for computing the set of all elements $\left(h_{1}, \ldots, h_{s}\right) \in$ $(K Q)^{s}$ such that $h_{1} f_{1}+\ldots+h_{s} f_{s}=0$ (resp. $f_{1} h_{1}+\ldots+f_{s} h_{s}=0$ ) called left and right syzygies. This result will be very useful for instance for those who wish to study the intersection of ideals since syzygies play a central role in finding a generating set of the intersection of two ideals.

\section{Preliminaries}

Definition 1.1. By a directed graph or a quiver we mean a quadruple $\Gamma=\left(\Gamma^{0}, \Gamma^{1}, r, s\right)$ where $\Gamma^{0}$ is the set of vertices, $\Gamma^{1}$ the set of edges and $r s: \Gamma^{1} \rightarrow \Gamma^{0}$ are maps. If $e \in \Gamma^{1}$ is an edge, then $s(e)$ the is called source of $e$ and $r(e)$ is the range of $e$. A sequence of edges $\alpha=e_{1} \ldots e_{n}$ such that $r\left(e_{i}\right)=s\left(e_{i+1}\right)$ for $i=1, \ldots, n-1$ is called path in $\Gamma$. In this case we denote $s(\alpha)=s\left(e_{1}\right)$ and $r(\alpha)=r\left(e_{n}\right)$. The number of edges in the path $\alpha$ denoted $L(\alpha)$ is called the length of $\alpha$. A vertex is regarded as a path of length zero. A closed path is a path $\alpha$ such that $s(\alpha)=$ $r(\alpha)$. A cycle is a closed path $\alpha$ such that if $e_{i}$ and $e_{j}$ are edges occurring in $\alpha$ then $s\left(e_{i}\right) \neq s\left(e_{j}\right) \forall i \neq j$. We 
denote by path ( $\Gamma)$ the set of all paths in $\Gamma$. If $\alpha$ and $\beta$ are paths, then we define the multiplication as follow: $\alpha \beta$ is the path adjoining $\alpha$ and $\beta$ by concatenation if $r(\alpha)=s(\beta)$; otherwise we get zero.

Let $K$ be a field and $\Gamma$ be a quiver. The set of all linear combinations of paths in $\Gamma$ with coefficients in $K$ together with the above multiplication is called path $K$-algebra. In the other hand, if $\Gamma=\left(\Gamma^{0}, \Gamma^{1}, r, s\right)$ is a quiver, then the path $K$-algebra $K \Gamma$ is the free associative algebra $K<\Gamma^{0} \cup \Gamma^{1}>$ generated by $\Gamma^{0} \cup \Gamma^{1}$ satisfying to: $v_{i} v_{j}$ $=\delta_{i j} v_{i} \forall v_{i} v_{j} \in \Gamma^{0}$ and $s(e) e=e r(e)=e \forall e \in \Gamma^{1}$.

A quiver $\Gamma$ if row-finite if $\forall e \in \Gamma^{1}$, we have $s^{-1}(e)<\infty$.

Remark 1.2. If $f \in K \Gamma$ then $f=\sum_{i} a_{i} p_{i}$ where $a_{i} \in K, p_{i} \in$ path $(\Gamma)$ and only finitely many $a_{i}=0$. Elements of $K \Gamma$ will be called polynomials and paths will be called monomials. Since the path algebra is unital, then in this work we will always consider $1_{K \Gamma}=\sum_{v \in \Gamma^{0}} v$.

Definition 1.3. - A well-ordering in path $(\Gamma)$ is a total ordering with the condition that every subset of path $(\Gamma)$ has a least element.

A well-ordering < is called left-admissible in path ( $\Gamma)$ if $\forall p, q, r \in$ path$(\Gamma)$, we have $p<q \Rightarrow r p<r q$ whenever $r p$ and $r q$ are both non-zeros.

A well-ordering < is called right-admissible in path ( $\Gamma)$ if $\forall p, q, s \in$ path $(\Gamma)$, we have $p<q \Rightarrow p s<q s$ whenever $p s$ and $q s$ are both non-zeros.

Example 1.4. - left-lexicographic order

Let $p=e_{1} \cdots e_{r}$ and $q=f_{1} \cdots f_{l}$ be two paths in $\Gamma$. We say that $p$ is less than $q$ with respect to the left-lexicographic order and we denote $p<$ llex $q$ if there exists a path $m$ (otherwise we set $m=1$ ) such that $p=m e_{k} \cdots e_{r}, q=m f_{s} \cdots f_{l}$ and $e_{k}<f_{s}$.

Remark 1.5. The left lexicographic order is not a left-admissible ordering since it is not a well-ordering. For example, for the following graph

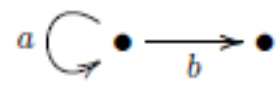

with $a<b$ we have $a b>_{\text {llex }} a^{2} b>_{\text {Ilex }}>a^{3} b>_{\text {Ilex }} \cdots$ then the subset $\left\{a^{n} b / n \in \mathrm{N} \backslash\{0\}\right\} \subset$ path $(\Gamma)$ doesn't have a least element.

\section{length left-lexicographic order}

Let $p=e_{1} \cdots e_{r}$ and $q=f_{1} \cdots f_{l}$ be two paths in $\Gamma$. We say that $p$ is less than $q$ with respect to the length left-lexicographic order and we denote $p<$ Lex $q$ if $L(p)<L(q)$ or $L(p)=L(q)$ and $p<$ llex $q$.

Remark 1.6. The length left-lexicographic order is a left-admissible ordering.

right-lexicographic order

Let $p=e_{1} \cdots e_{r}$ and $q=f_{1} \cdots f_{l}$ be two paths in $\Gamma$. We say that $p$ is less than $q$ with respect to the right-lexicographic order and we denote $p<_{\text {rlex }} q$ if there exists a path $m$ (otherwise we set $m=1$ ) such that $p=e_{1} \cdots e_{k} m, q=f_{1} \cdots f_{s} m$ and $e_{k}<f_{s}$.

Remark 1.7. The right lexicographic order is not a right-admissible ordering since it is not a well-ordering. For example, for the following graph

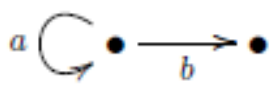

with $a<b$ we have $b a>_{\text {rlex }} b a^{2}>_{\text {rlex }}>b a^{3}>_{\text {rlex }} \cdots$ then the subset $\left\{b a^{n} / n \in \mathrm{N} \backslash\{0\}\right\} \subset$ path $(\Gamma)$ doesn't have a least element.

\section{length right-lexicographic order}

Let $p=e_{1} \cdots e_{r}$ and $q=f_{1} \cdots f_{l}$ be two paths in $\Gamma$. We say that $p$ is less than $q$ with respect to the length right-lexicographic order and we denote $p<_{\text {rLex }} q$ if $L(p)<L(q)$ or $L(p)=L(q)$ and $p<_{\text {rlex }} q$.

Remark 1.8. The length right-lexicographic order is a right-admissible ordering. 
Definition 1.9. Let $<$ be a left or right admissible ordering and $f \in K \Gamma \backslash\{0\}$. Then we call:

- the leading monomial of $f$ denoted $\operatorname{Lm}(f)$ to be the biggest monomial occurring to $f$ with respect to <;

- the leading coecient of $f$ denoted $L c(f)$ to be the coecient of $\operatorname{Lm}(f)$ in $f$;

the leading term of $f$ denoted $\operatorname{Lt}(f)=\operatorname{Lc}(f) \operatorname{Lm}(f)$.

In what follow we denote by $R=K \Gamma$.

Definition 1.10. (1) A subset of $I_{L}$ of $K \Gamma$ is called left ideal if the following conditions hold:

- $0 \in I_{L}$;

- $f+g \in I_{L} \forall f, g \in I_{L}$ :

- $f h \in I_{L} \forall f \in R$ and $h \in I_{L}$.

(2) A subset of $I_{R}$ of $K \Gamma$ is called right ideal if the following conditions hold:

- $0 \in I_{R}$ :

- $f+g \in I_{R} \forall f, g \in I_{R}$ :

- $f h \in I_{R} \forall f \in R$ and $h \in I_{R}$.

Remark 1.11. If $E$ is a subset of $R$, then we denote by $\langle E\rangle_{L}=\left\{\sum_{i} h_{i} g_{i} / h_{i} \in R \quad g_{i} \in E \forall i\right\}$ the left ideal generated by $E$ and $\left\langle E>_{R}=\left\{\sum_{i}\right.\right.$ gifi $/ g_{i} \in E$ and $\left.f_{i} \in R \forall i\right\}$ the right ideal generated by $E$. We denote by $\operatorname{Lm}(E)_{L}=<L m(f) / f \in E \backslash\{0\}>_{L}$ and $\operatorname{Lm}(E)_{R}=<L m(f) / f \in E \backslash\{0\}>_{R}$.

Definition 1.12. A left (respectively right) ideal of a path $K$-algebra $K \Gamma$ is called left (respectively right) monomial if it is generated by monomials.

Remark 1.13. A left (respectively right) monomial ideal is also called left (respectively right) path ideal.

\section{Left Groebner Bases on $K Q$}

In this section we denote by $Q$ a quiver and $R=K Q$ a path $K$-algebra. The goal of this section is to propose a method for computing a left Groebner basis of a left ideal of $R=K Q$. By division in this section we will always mean on the left i.e $a \mid b$ if there exists $c$ such that $b=c \cdot a$.

Definition 2.1. Let $I_{L}$ be a left ideal of $R$ and $G$ be a subset of $I_{L}$. We say that $G$ is a left Groebner basis for $I_{L}$ with respect to a given left-admissible ordering if for every $f \in I_{L}$, there exists $g \in G$ such that $\operatorname{Lm}(g)$ divides $\operatorname{Lm}(f)$ i.e there exists $h \in R$ such that $\operatorname{Lm}(f)=\operatorname{Lm}(h) \operatorname{Lm}(g)$. In the other hand, $G$ is a left Groebner basis for $I_{L}$ if $\operatorname{Lm}\left(I_{L}\right)_{L}=\operatorname{Lm}(G)_{L}$.

Remark 2.2. A left Groebner basis is a generating set of a left ideal, since the path $K$-algebra $R$ of a given graph is not necessarily left noetherian, for example for the graph

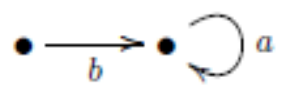

The corresponding path $K$-algebra is not left noetherian since the left ideal $h b a^{n} / n \in \mathrm{N} \backslash\{0\} \mathrm{i}_{L}$ is not finitely generated. a left Groebner basis may be innite and in this case the computation won't be interesting. In this work we will deal only with noetherian path $K$-algebras.

Definition 2.3. Let $Q$ be a quiver. A cycle $c$ in $Q$ has an entry if there exists an edge in $Q$ not occurring in $c$ with its range in $c$. For example the cycle of the graph of the Remark 2.2 has an entry.

Proposition 2.4. The path $K$-algebra $K Q$ is left noetherian if and only if no cycle in $Q$ has an entry.

Proof. Assume that in $Q$ there exists a cycle with an entry, we denote that cycle by $c$. Let $p$ be a path in $Q$ not occurring in $c$ with $r(p)$ a vertex of $c$. Then the ideal $\left\langle p c, p c^{2}, p c^{3}, \cdots>_{L}\right.$ is not finitely generated. Thus $K Q$ is not left noetherian. Conversely, if no cycle has an entry then, we have two possibilities: either $Q$ contains no cycle or it contains nitely many cycles (since the graph is row-nite) with no entry.

- If $Q$ contains no cycle then we have a nite number of paths, thus any left ideal is nitely generated.

- Assume that $Q$ contains nitely many cycles with no entry. Since $Q$ contains only nitely many paths and 
cycles, if there exists a left ideal which is not finitely generated, it must contains non-zero paths of the form $q c^{n}$ where $q$ is a path and $c$ is a cycle with $n \in \mathrm{N}$. Such paths are defined if $r(q)$ occurs in $c$, that is $c$ must have an entry. Contradicting the hypothesis.

In the rest of this section, $R=K Q$ will be considered to be a left noetherian path $K$-algebra.

Proposition 2.5. Let $R$ be a left noetherian path $K$-algebra and < be left a admissible ordering.

1) A path $p \in T=<q_{i} / q_{i} \in$ path $(Q) \forall 1 \leq i \leq r>_{L}$ i there exists $i_{0}$ such that $q_{i_{0}}$ divides $p$.

2) If $I_{L} \subset R$ is a left monomial (path) ideal then $I_{L}$ has a unique finite minimal path generating set.

Proof. (1) Straightforward.

Let $A$ be the set of all paths in $I_{L}$ and let $M \subset A$ be the set of minimal (by division) paths i.e $M=\{p \in A$ /if $q$ $\in A$ divides $p$ then $q=p\}$. We want to prove that $M$ is the unique nite minimal path generating set of $I_{L}$.

Since $M \subset A$ then $\left\langle M>_{L} \subseteq I_{L}\right.$.

Let $p \in I_{L}$ be a path, then $p \in A$. Let $B$ be the set of all paths that generates $I_{L}$ i.e $I_{L}=\left\langle B>_{L}\right.$. If $p$ is not minimal (i.e $p \notin M$ ) then $p=a b$ where $a \in \operatorname{path}(Q)$ and $b \in B$.

- If $b$ is minimal (i.e $b \in M$ ) then $p \in\left\langle M>_{L}\right.$.

- If $b$ is not minimal (i.e $b \notin M$ ) then by induction $b$ is multiple of an element of $M$, since < is a well-ordering. Thus $p \in\left\langle M>_{L}\right.$ and $I_{L}=\left\langle M>_{L}\right.$. Since $R$ is left noetherian then $I_{L}=\langle M\rangle_{L}$ is finitely generated. Thus $M$ is finite.

Let $M^{\prime}$ be a minimal finite paths generating set of $\mathrm{I}_{\mathrm{L}}$. Let us prove that $M=M^{\prime}$. Since $M^{\prime}$ is minimal then $M^{\prime} \subseteq M$. Let $m \in M \subset<M>_{L}=I_{L}=\left\langle M^{\prime}>_{L}\right.$ then there exists $m^{\prime} \in M^{\prime}$ such that $m^{\prime}$ divides $m$ which means by definition of $M$ that $m^{\prime}=m$.

2.1. Left division's algorithm. Let $F=\left\{f_{1}, \ldots, f_{s}\right\}$ be a set of elements of $R=K Q$ and $>$ be a left admissible ordering. Given element $g \in R \backslash\{0\}$, the following algorithm shows how to nd $q_{1}, \ldots, q_{s}, h \in K Q$ such that $g=q_{1} f_{1}+\cdots+q_{s} f_{s}+h$ satisfying.

A1 if $h \neq 0$, then each term occurring in $h$ is not divisible by any of $\operatorname{Lt}\left(f_{i}\right) \forall 1 \leq i \leq s$.

A2 $\operatorname{Lm}(g) \geq \operatorname{Lm}\left(q_{i} f_{i}\right)$ for each $i \in\{1, \ldots, s\}$ since $q_{i}$ are obtained from $g$.

Input: $f, f_{1}, \ldots, f_{s} \in F$ and a left admissible ordering $>=\left(>_{1},>_{2}\right)$.

Output: $q_{1}, \ldots, q_{s}, h \in F$ such that $f=q_{1} f_{1}+\cdots+q_{s} f_{s}+h$.

Initialization: $q_{1}:=q_{2}:=\cdots:=q_{s}:=h:=0$ and $v:=g$;

- While $v \neq 0$ do

$-i:=1$ :

- while $i \leq s$ do

* if $\operatorname{Lt}\left(f_{i}\right) \mid \operatorname{Lt}(v)$ then

$\cdot q_{i}:=q_{i}+\frac{\operatorname{Lt}(v)}{\operatorname{Lt}\left(f_{i}\right)}$

$\cdot v:=v-\frac{\operatorname{Lt}(v)}{\operatorname{Lt}\left(f_{i}\right)} f_{i}$;

* Else

$\cdot:=i+1:$

$-h:=h+\operatorname{Lt}(v)$

$-v:=v-L t(v)$

Proposition 2.6. Let $\left\{f_{1}, \ldots, f_{r}\right\} \subset R$ be a left Groebner basis for the ideal $I_{L}:=\left\langle f_{1}, \ldots, f_{r}\right\rangle_{L} \subset R$, $>$ be a left admissible ordering and $g \in R \backslash\{0\} . g \in I_{L}$ if and only if the remainder $h$ of the division of $g$ by $f_{1}, \ldots, f_{r}$ is zero.

Proof. If $h=0$ then it is clear by the division's algorithm that $g \in I_{L}$. Conversely, if $g \in I_{L}$ then by the division's algorithm we can write $g=g_{1} f_{1}+\ldots+g_{r} f_{r}+h$ for some $g_{1}, \ldots, g_{r}, h \in R$. Observe that $h \in I_{L}=\Rightarrow m(h) \in$ $\operatorname{Lm}\left(I_{L}\right)=<\operatorname{Lm}\left(f_{1}\right), \ldots, \operatorname{Lm}\left(f_{r}\right)>$ which is impossible by A1). 
Definition 2.7. (Left useful paths)

Let $p, q \in \operatorname{path}(Q)$ be paths in $Q$. Two paths $u, v$ are called left useful for $p$ and $q$ with respect to the left admissible ordering <, if $u p=v q \neq 0$ and for any other paths $w_{1}$ and $w_{2}$ satisfying $w_{1} p=w_{2} q \neq 0$, we have $u$ divides $w_{1}$ and $v$ divides $w_{2}$ ( both on the left).

Definition 2.8. (Left S-polynomial)

Let $f, g$ be non-zero polynomials and < be a left admissible ordering. Let $u, v$ be left useful paths for $\operatorname{Lm}(f)$ and $\operatorname{Lm}(g)$ (i.e $u \operatorname{Lm}(f)=v \operatorname{Lm}(g)$ ), then the left S-polynomial $S_{L}(f, g)$ of $f$ and $g$ is dened as

$$
S_{L}(f, g)=\frac{u}{L c(f)} f-\frac{v}{L c(g)} g .
$$

Remark 2.9. If there exist no left useful paths for $\operatorname{Lm}(f)$ and $\operatorname{Lm}(g)$, we claim that $S_{L}(f, g)=0$.

Definition 2.10. (Left extended S-Polynomial)

Let $f \in R$ be a non-zero polynomial and $>$ be a left admissible ordering. We define the left extended S-polynomial $S_{L}(f)$ of $f$ as $S_{L}(f)=p \cdot f$ where $p$ is a path in $K Q$ such that $p \cdot \operatorname{Lm}(f)=0$ and $p \cdot f \neq 0$.

Remark 2.11. Let $p$ and $q$ be two paths satisfying the conditions of the Definition 3.9, if $q$ divides $p$ then we choose $S_{L}(f)=q \cdot f$.

Definition 2.12. Let $I_{L}$ be a left path ideal and $w \in \operatorname{path}(Q)$. We define the left quotient ideal of $I_{L}$ and $w$ by $I_{L}: w=\left\{p \in \operatorname{path}(Q) / p \cdot w \in I_{L}\right\}$.

Lemma 2.13. Let $I_{L}=\left\langle w_{1}, \ldots, w_{r}\right\rangle_{L}$ be a left path ideal of $R, t$ be a monomial and $>$ be a left admissible ordering. Then the quotient ideal $I_{L}: t=\left\{p\right.$ monomial in $\left.R / p t \in I_{L}\right\}$ can be written as

$$
I_{L}: t=\left\langle v_{1}, \ldots, v_{l}\right\rangle_{L}
$$

where for each $1 \leq i \leq l \leq r$ there exists a monomial $p_{i}$ such that $v_{i} t=p_{i} w_{i}$ and $v_{i} p_{i}$ are left useful for $t$ and $w_{i}$.

Proof. Let $x \in\left\langle v_{1}, \ldots, v_{r}\right\rangle_{L}$ then there exist $i \leq r$ and a path $y \in R$ such that $x=y v_{i}$. Our goal is to show that $x t$ $\in I_{L}$. By hypothesis there is a path $p_{i}$ such that $v_{i} t=p_{i} w_{i}$ and $v_{i} p_{i}$ are left useful for $t, w_{i}$. Multiplying by $y$ we get $y v_{i} t=y p_{i} w_{i}$ i.e $x t=y p_{i} w_{i} \in I_{L}$ thus $x \in I_{L}: t$.

Conversely let $x \in I_{L}: t$ then $x \cdot t \in I_{L}$, that is, there exist $i \leq r$ and a path $y \in R$ such that $x t=y w_{i}$, this means there exist left useful paths $x^{\prime}, y^{\prime}$ for $t, w_{i}$ such that $x^{\prime} \mid x$ and $y^{\prime} \mid y$. Set $v_{i}=x^{\prime}$ then $x \in\left\langle v_{1}, \ldots, v_{r}\right\rangle$.

Definition 2.14. Let $R=K Q$ and $T=R$ se a let a left free $R$-module with basis $\left\{e_{1}, \ldots, e_{s}\right\}$. By a monomial in $T$ involving the component $e_{j}$ we mean a monomial in $R$ times $e_{j}$ that is $p e_{j}$ where $p$ is a monomial in $R$.

Definition 2.15. (Syzygy)

Let $T=R^{s}$ be a left free $R$-module with basis $\left\{e_{1}, \ldots, e_{s}\right\}$ and let $I_{L}=\left\langle f_{1}, \ldots, f_{s}>_{L}\right.$ be a left ideal of $R$. By a left syzygy we mean an element of the kernel of the $R$-module homomorphism

$$
\begin{aligned}
\psi: T=R^{s} & \rightarrow R \\
e_{i} & \mapsto f_{i} .
\end{aligned}
$$

We call $\operatorname{ker}(\psi)$ the (first) left syzygy module on $f_{1}, \ldots, f_{s}$ written $\operatorname{syz}\left(f_{1}, \ldots, f_{s}\right)_{L}=\operatorname{ker}(\psi)$.

Definition 2.16. (Induced ordering)

Let $G=\left\{f_{1}, \ldots, f_{s}\right\}$ be a set of polynomials and $>$ be a left admissible ordering. Let $T=R^{s}$ be a left free $R$-module with basis $\left\{e_{1}, \ldots, e_{s}\right\}$. We define the module ordering $>1$ induced by $G$ and $>$ as follow: if $p, q$ are two paths in $R$ then

$$
\begin{aligned}
& p e_{i}>_{1} q e_{j} \Leftrightarrow p \operatorname{Lm}\left(f_{i}\right)>q \operatorname{Lm}\left(f_{j}\right)\left(\text { whenever } p \operatorname{Lm}\left(f_{i}\right) \neq 0\right. \\
& \left.\neq q \operatorname{Lm}\left(f_{j}\right)\right) \text { or } \\
& 0 \neq p \operatorname{Lm}\left(f_{i}\right)=q \operatorname{Lm}\left(f_{j}\right) \text { and } i> \\
& \text { j. }
\end{aligned}
$$


Theorem 2.17. (Left Buchberger's criterion)

Let $f_{1}, \ldots, f_{s} \in R$ be non-zero polynomials and $>$ be a left admissible ordering on $R$. Then $\left\{f_{1}, \ldots, f_{s}\right\}$ form a left-Groebner basis for $I_{L}=\left\langle f_{1}, \ldots, f_{s}\right\rangle$ if and only if the following conditions are satisfied:

1) For each $i>j$, the remainder of $S_{L}\left(f_{i} f_{j}\right)$ on division's algorithm by $\left\{f_{1}, \ldots, f_{s}\right\}$ is zero.

2) For each $i$, the remainder of $S_{L}\left(f_{i}\right)$ on division's algorithm by $\left\{f_{1}, \ldots, f_{s}\right\}$ is zero.

\section{Proof of the Left Buchberger's Criterion}

Proof. If $\left\{f_{1}, \ldots, f_{S}\right\}$ form a left Groebner basis for $I_{L}=\left\langle f_{1}, \ldots, f_{s}\right\rangle$ then by the Proposition 2.6 and using the left division's algorithm we have for $k<i, S_{L}\left(f_{i} f_{k}\right)=\sum_{i=1}^{S} g^{i} f_{i}+0$ and for each $i, S_{L}\left(f_{i}\right)=\sum_{i=1}^{S} h^{i} f_{i}+0$ since $S_{L}\left(f_{i}\right)$, $S_{L}\left(f_{i} f_{k}\right) \in I_{L}$. and by proposition 2.6 .

Conversely, suppose that for $1 \leq k<i \leq s$ all the remainders of $S_{L}\left(f_{i} f_{k}\right)$ under the left division's algorithm by $\left\{f_{1}, \ldots, f_{s}\right\}$ are zero and for each $i$, all the remainders of the $S_{L}\left(f_{i}\right)$ under the left division's algorithm by $\left\{f_{1}, \ldots, f_{s}\right\}$ are zero. We have for

$$
i>k, S_{L}\left(f_{i}, f_{k}\right)=\sum_{j=1}^{s} g_{j}^{i k} f_{j} \text { with } \operatorname{Lm}\left(S_{L}\left(f_{i}, f_{k}\right)\right) \geq \operatorname{Lm}\left(g_{j}^{i k} f_{j}\right) \quad \text { (**). }
$$

Applying the definition of the left S-polynomial we have

$$
\frac{v}{L c\left(f_{i}\right)} f_{i}-\frac{w}{L c\left(f_{k}\right)} f_{k}=\sum_{j=1}^{s} g_{j}^{i k} f_{j}
$$

where $v, w$ are left useful paths for $\operatorname{Lm}\left(f_{i}\right)$ and $\operatorname{Lm}\left(f_{k}\right)$. Observe that

$$
\begin{aligned}
& -g_{1}^{i k} f_{1}-\ldots-\left(g_{k}^{i k}+\frac{w}{L c\left(f_{k}\right)}\right) f_{k}-\ldots-\left(g_{i}^{i k}-\frac{v}{L c\left(f_{i}\right)}\right) f_{i}-\ldots-g_{s}^{i k} f_{s}=0, \text { this means that } \\
& \left(-g_{1}^{i k}, \ldots,-g_{k}^{i k}-\frac{w}{L c\left(f_{k}\right)}, \ldots,-g_{i}^{i k}+\frac{v}{L c\left(f_{i}\right)}, \ldots,-g_{s}^{i k}\right) \text { is a syzygy for } f_{1}, \ldots, f_{s .} \text { Set } \\
& G_{i k}=\left(-g_{1}^{i k}, \ldots,-g_{k}^{i k}-\frac{w}{L c\left(f_{k}\right)}, \ldots,-g_{i}^{i k}+\frac{v}{L c\left(f_{i}\right)}, \ldots,-g_{s}^{i k}\right)
\end{aligned}
$$

By $\left(^{*}\right)$ and the definition of the left S-polynomial we can notice that $\operatorname{Lm}\left(g_{j}^{i k} f_{j}\right) \leq \operatorname{Lm}\left(S_{L}\left(f_{i} f_{k}\right)\right)<v \operatorname{Lm}\left(f_{i}\right)=$ $w \operatorname{Lm}\left(f_{k}\right)$, with respect to the induced ordering, this means that $v e_{i}>w e_{k}$ since $i>k$, this leads us to

$\operatorname{Lm}\left(G_{i k}\right)=v_{i}(2)$.

In the other hand, we have for some $i, S_{L}\left(f_{i}\right)=p_{i} f_{i}$ where $p_{i}$ is a vertex such that $p_{i} \operatorname{Lm}\left(f_{i}\right)=0$ and $p_{i} f_{i} \neq 0$. By the division's algorithm we have $S_{L}\left(f_{i}\right)=\sum_{j=1}^{S} h_{j}^{i} f_{j}=p_{i} f_{i}$, this means that

$$
-h_{1}^{i} f_{1}-\ldots-\left(-h_{i}^{i}-p_{i}\right) f_{i}-\ldots-h_{s}^{i} f_{s}=0,
$$

that is $H_{i}=\left(-h_{1}^{i}, \ldots,-\left(-h_{i}^{i}-p_{i}\right), \ldots,-h_{s}^{i}\right)$ is a left syzygy for $f_{1}, \ldots, f_{s}$.

Observe that $\operatorname{Lm}\left(h^{i}\right) \operatorname{Lm}\left(f_{j}\right) \leq \operatorname{Lm}\left(S_{L}\left(f_{i}\right)\right)=\operatorname{Lm}\left(p_{i} f_{i}\right)=p_{i} \operatorname{Lm}\left(t_{i}\right)$ where $t_{i}$ is a polynomial occurring in $f_{i}$ such that $p_{i} f_{i}=p_{i} t_{i}$. By the Schreyer's ordering induced by $>$ and $\left\{f_{1}, \ldots, f_{i-1}, t_{i} f_{i+1}, \ldots, f_{s}\right\}$ we have $\operatorname{Lm}\left(H_{i}\right)=p_{i} e_{i}$.

Let us now prove that $\left\{f_{1}, \ldots, f_{s}\right\}$ form a left Groebner basis for $I_{L}$.

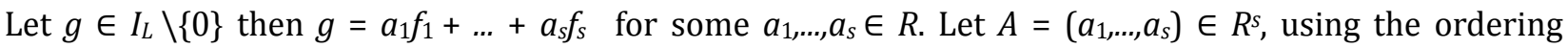
induced by $>$ and $\left\{f_{1}, \ldots, f_{s}\right\}$, we can extend the left division's algorithm in $R^{s}$. Let $G=<g_{1}, \ldots, g_{s}>\in R^{s}$ be the remainder under the left division's algorithm of $A$ by the set of all non-zero $G_{i j}$ and $H_{i}$ (listed in some order), then we have the expression.

$\mathrm{A}=\sum_{G_{I J \neq 0}} q_{i} G_{i j}+\sum_{H_{i} \neq 0} p_{i} H_{i}+\mathrm{G}(\mathrm{C})$.

Let $F=\left(f_{1}, \ldots, f_{r}\right)^{t}$, by multiplying $(C)$ by $F$ we get

$\mathrm{AF}=\mathrm{g}=\mathrm{a} 1 \mathrm{f} 1+\ldots+\mathrm{asfs}=\mathrm{g} 1 \mathrm{f} 1+\ldots+\mathrm{gsfs}(\mathrm{D})$.

We transform $(D)$ as follow:

$$
g=\sum_{i=1}^{S} \operatorname{Lt}\left(g_{i}\right) \operatorname{Lt}\left(f_{i}\right)+\sum_{i=1}^{S} \operatorname{tail}\left(g_{i}\right) \operatorname{Lt}\left(f_{i}\right)+\sum_{i=1}^{S} g_{i} \operatorname{tail}\left(f_{i}\right) \quad \text { Where } \operatorname{tail}\left(f_{i}\right)=f_{i}-\operatorname{Lt}\left(f_{i}\right)
$$


1) If $\operatorname{Lm}(g) \neq \operatorname{Lm}\left(\sum_{i=1}^{s} g_{i} \operatorname{tail}\left(f_{i}\right)\right)$ then it is clear that

$$
\operatorname{Lm}(g)=\operatorname{Lm}\left(\sum_{i=1}^{S} \operatorname{Lt}\left(g_{i}\right) \operatorname{Lt}\left(f_{i}\right)+\sum_{i=1}^{S} \operatorname{tail}\left(g_{i}\right) \operatorname{Lt}\left(f_{i}\right)\right.
$$

that is $\operatorname{Lm}(g) \in<\operatorname{Lm}\left(f_{1}\right), \ldots, \operatorname{Lm}\left(f_{s}\right)>$ and by the Lemma 2.13 There exists $k \in\{1, \ldots, s\}$ such that $\operatorname{Lm}\left(f_{k}\right)$ | $\operatorname{Lm}(g)$.

2) If $\operatorname{Lm}(\mathrm{g})=\operatorname{Lm}\left(\sum_{i=1}^{s} g_{i} \operatorname{tail}\left(f_{i}\right)\right)$ then there exists $\mathrm{K} \subseteq\{1, \ldots, \mathrm{s}\}$ such that

$$
\sum_{i \in K} \operatorname{Lt}\left(g_{i}\right) \operatorname{Lt}\left(f_{i}\right)=0 \quad \text { and } \quad \sum_{i \in K} \operatorname{Lt}\left(g_{i}\right) \operatorname{tail}\left(f_{i}\right) \neq 0 \text {. }
$$

If there exists $j \in K$ such that $\operatorname{Lm}\left(g_{j}\right) \operatorname{Lm}\left(f_{j}\right) \neq 0$ then without loss of generalities we can assume that $K=$ $\left\{i_{1}, \ldots, i_{r}\right\}$ and $j=i_{r}$, then

$$
\operatorname{Lm}\left(g_{j}\right) \operatorname{Lm}\left(f_{j}\right)=-\operatorname{Lm}\left(g_{i 1}\right) \operatorname{Lm}\left(f_{i 1}\right)-\ldots-\operatorname{Lm}\left(g_{j^{-1}}\right) \operatorname{Lm}\left(f_{j^{-1}}\right)
$$

that is $\operatorname{Lm}\left(g_{j}\right) \operatorname{Lm}\left(f_{j}\right) \in<\operatorname{Lm}\left(f_{i 1}\right), \ldots, \operatorname{Lm}\left(f_{j-1}\right)>\quad$ and $\quad \operatorname{Lm}\left(g_{j}\right) \in<\operatorname{Lm}\left(f_{i 1}\right), \ldots, \operatorname{Lm}\left(f_{j-1}\right)>: \operatorname{Lm}\left(f_{j}\right)$, by the Lemma 2.13 we have $\operatorname{Lm}\left(g_{j}\right) \in\left\langle v_{i 1}, \ldots, v_{j-1}\right\rangle$ where for $i_{1} \leq l \leq j-1$ we have $v_{l} \operatorname{Lm}\left(f_{j}\right)=p_{l} \operatorname{Lm}\left(f_{l}\right)$ and $v_{l} p_{l}$ are left useful monomials for $\operatorname{Lm}\left(f_{j}\right)$ and $\operatorname{Lm}\left(f_{l}\right)$. By the Lemma 2.13 there exists $k \in K$ such that $v_{k} \mid \operatorname{Lm}\left(g_{j}\right)$ that is $\operatorname{Lm}\left(G_{k j}\right) \mid$ $\operatorname{Lm}\left(g_{j}\right) e_{j}$ which contradict the fact that no monomial occurring in the remainder $G$ is divisible by any of the $\operatorname{Lm}\left(G_{i j}\right)$.

Assume that $\operatorname{Lm}\left(g_{j}\right) \operatorname{Lm}\left(f_{j}\right)=0 \forall j \in K$ then $S_{L}\left(f_{j}\right)=p_{j} f_{j}$ where for each $j, p_{j}$ is a vertex such that $p_{j} \neq s\left(\operatorname{Lm}\left(f_{j}\right)\right)$. Choose $p_{j}=r\left(\operatorname{Lm}\left(g_{j}\right)\right)$ then We have $\operatorname{Lm}\left(g_{j}\right) e_{j}=\operatorname{Lm}\left(g_{j}\right) p_{j} e_{j}=\operatorname{Lm}\left(g_{j}\right) \operatorname{Lm}\left(H_{j}\right)$, this means that $\operatorname{Lm}\left(H_{j}\right)$ divides $\operatorname{Lm}\left(g_{j}\right) e_{j}$ on the left, this is a contradiction since no monomial occurring in the remainder $G=\left(g_{1}, \ldots, g_{s}\right)$ is divisible by any of $\operatorname{Lm}\left(H_{j}\right)$.

Remark 2.18. To compute a left Groebner basis for $\left.I_{L}=<f_{1}, \ldots, f_{S}\right\rangle_{L}$ like in the Theorem 3.16, there is no need to consider all the left S-polynomials $S_{L}\left(f_{i}, f_{j}\right)=\frac{v_{j i}}{L c\left(f_{i}\right)} f_{i}-\frac{v_{j i}}{L c\left(f_{j}\right)} f_{j}$ for some paths $v_{j i}, v_{i j}$ left useful to $\operatorname{Lm}\left(f_{i}\right)$ and $\operatorname{Lm}\left(f_{j}\right)$. It is straightforward to see that

$$
S_{L}\left(f_{i}, f_{j}\right)=-S_{L}\left(f_{j}, f_{i}\right) \forall i, j
$$

so instead of considering all the left S-polynomials, we can just consider left the S-polynomials $S_{L}\left(f_{i} f_{j}\right)$ with $j$ $<i$. We can even do better by using the following technique.

For $i=2,3, \ldots, s$, consider the left path ideal

$M_{i}=\left\langle\operatorname{Lm}\left(f_{1}\right), \ldots, L m\left(f_{i-1}\right)\right\rangle: \operatorname{Lm}\left(f_{i}\right)=\left\langle v_{1 i}, \ldots, v_{i-1 i}\right\rangle$ where $v_{j i} \forall 1 \leq j \leq i-1$, are like in the Proposition 2.13 i.e for each $v_{j i} \neq 0$, there exists a path $v_{i j}$ such that $v_{j i}$ and $v_{i j}$ are left useful for $\operatorname{Lm}\left(f_{i}\right), \operatorname{Lm}\left(f_{j}\right)$. In the other words, each $v_{j i} \neq 0$ corresponds to a left S-polynomial $S_{L}\left(f_{i}, f_{j}\right)=\frac{v_{j i}}{L c\left(f_{i}\right)} f_{i}-\frac{v_{j i}}{L c\left(f_{j}\right)} f_{j}$.

As it turn out, there is no need to consider all the generators $v_{j i}$ of $M_{i}$ (i.e there is no need to consider all the corresponding S-polynomials of each $v_{j i}$ in in the Buchberger's criterion. For each $i$, and for each minimal path generator $v_{j i}$ of $M_{i}$, consider only the corresponding left S-polynomial $S_{L}\left(f_{i} f_{j}\right)$, and compute the compute the remainder $h_{i j}$ of the division of $S_{L}\left(f_{i} f_{j}\right)$ by $f_{1}, \ldots, f_{s}$.

Input: an ideal $I_{L}=\left\langle f_{1}, \ldots, f_{S}\right\rangle \subset R$ and a left admissible order $>$ on $R$.

Output: a left Groebner basis for $I_{L}$. 
A) Set $k=s$.

B) For $i=2, \ldots, k$, and for each minimal path generator $v_{j i}$ of

$$
M_{i}=\left\langle\operatorname{Lm}\left(f_{1}\right), \ldots, \operatorname{Lm}\left(f_{i-1}\right)\right\rangle_{L}: \operatorname{Lm}\left(f_{i}\right) \subset R,
$$

compute a remainder $h_{i j}$ as described in the Remark 2.18 above.

C) If some $h_{i j}$ is non-zero, set $k=k+1, f_{k}=h_{i j}$, and go back to B).

D) Return $\left\{f_{1}, \ldots, f_{k}\right\}$.

Example 2.19. For the graph $\Gamma$

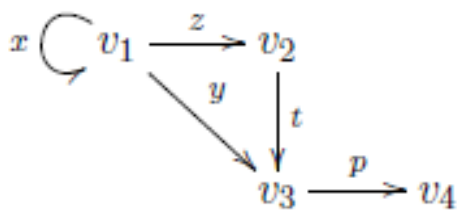

Let us compute the left Groebner bases for $F=\left\{f_{1}=z t p+y p, f_{2}=x^{2} z t+y p, f_{3}=y p+z t\right\}$ in $R=\mathbb{Q} \Gamma$ with $x>$ llex $y>$ llex $Z>$ llex $t>$ llex $p>$ llex $v 1>$ llex $v 2>$ llex $v 3>$ llex $v 4$ where by $>$ llex we mean the left length lexicographic ordering.

It is easy to see that $\operatorname{Lm}\left(f_{1}\right)=z t p, \operatorname{Lm}\left(f_{2}\right)=x^{2} z t, \operatorname{Lm}\left(f_{3}\right)=y p$.

- For $i=2$, it is easy to see that $\left\langle z t p>_{L}: x^{2} z t=0\right.$ i.e there are no useful monomials between $\operatorname{Lm}\left(f_{1}\right)$ and $\operatorname{Lm}\left(f_{2}\right)$, we claim that $S\left(f_{2} f_{1}\right)=0$.

- For $i=3$, it is easy to see that $\left\langle z t p, x^{2} z t>_{L}: y p=0\right.$ i.e there are no useful monomials between $\operatorname{Lm}\left(f_{1}\right)$ and $\operatorname{Lm}\left(f_{3}\right)$, and between $\operatorname{Lm}\left(f_{2}\right)$ and $\operatorname{Lm}\left(f_{3}\right)$. We claim that $S_{L}\left(f_{3}, f_{1}\right)=S\left(f_{3}, f_{2}\right)_{L}=0$. Observe that $S_{L}\left(f_{1}\right)=$ $S_{L}\left(f_{2}\right)=S_{L}\left(f_{3}\right)=0$.

Thus the set $F=\left\{f_{1}=z t p+y p, f_{2}=x^{2} z t+y p, f_{3}=y p+z t\right\}$ is a left Groebner basis for $I_{L}=\langle F\rangle_{L}$.

With notations as in the left Buchberger's criterions above, we have the following theorem.

\section{Theorem 2.20. (Schreyer's theorem)}

If $\left\{f_{1}, \ldots, f_{S}\right\}$ is a left Groebner basis for $I_{L}=\left\langle f_{1}, \ldots, f_{S}\right\rangle_{L}$ w.r.t the an admissible ordering >, then the set $T=\left\{G_{i j}\right.$, $\left.H_{i} \in \operatorname{syz}_{L}\left(f_{1}, \ldots, f_{S}\right) / G_{i j} \neq 0 \neq H_{i}\right\}$ considered in the proof of the left Buchberger's criterion form a left Groebner basis for $\operatorname{syz}_{L}\left(f_{1}, \ldots, f_{S}\right)$ w.r.t the ordering $>_{1}$ induced by $>$ and $\left\{f_{1}, \ldots, f_{S}\right\}$.

Proof. Let $A \in \operatorname{Syz}_{L}\left(f_{1}, \ldots, f_{S}\right)$, we wish to prove that $\exists h \in T$ such that $L t(h) \mid \operatorname{Lt}(A)$.

By the left division's algorithm in $R^{s}$ for $A$ by $T$ we have

$$
A=\sum_{i j} Q_{i j} G_{i j}+\sum_{i} p_{i} H_{i}+G
$$

where $Q_{i j}, H_{i} \in R$ and $G=\left(g_{1}, \ldots, g_{s}\right) \in R^{s}$. Let $F=\left(f_{1}, \ldots, f_{s}\right)^{t} \in R^{s}$, by multiplying $\quad(*) \quad$ by $F$ we get

$$
g_{1} f_{1}+\ldots+g_{s} f_{s}=0(2 *)
$$

Assume that $G=\left(g_{1}, \ldots, g_{s}\right)$ is a non-trivial syzygy, then there exists $K=\left\{i_{1}, \ldots, i_{r}\right\} \subset\{1, \ldots, s\}$ such that

$$
g_{i 1} f_{i 1}+\ldots+g_{i r} f_{i r}=0(3 *) .
$$

We can transform $(3 *)$ as follow

$$
\text { (4) }: 0=\sum_{i=i_{1}}^{i_{r}} \operatorname{Lt}\left(g_{i}\right) \operatorname{Lt}\left(f_{i}\right)+\sum_{i=i_{1}}^{i_{r}} \operatorname{tail}\left(g_{i}\right) \operatorname{Lt}\left(f_{i}\right)+\sum_{i=i_{1}}^{i_{r}} \operatorname{Lt}\left(g_{i}\right) \operatorname{tail}\left(f_{i}\right)
$$


It is clear that $\sum_{i=i_{1}}^{i_{r}} \operatorname{Lt}\left(g_{i}\right) \operatorname{Lt}\left(f_{i}\right)=0$

1) Assume that $\exists k \in K$ such that $\operatorname{Lm}\left(g_{k}\right) \operatorname{Lm}\left(f_{k}\right) \neq 0$. Without loss of generalities we can assume that $k=$ $i_{r}$, in this case

$$
\operatorname{Lt}\left(g_{i_{r}}\right) \operatorname{Lt}\left(f_{i_{r}}\right)=-\operatorname{Lt}\left(g_{i_{1}}\right) \operatorname{Lt}\left(f_{i_{1}}\right)-\cdots-\operatorname{Lt}\left(g_{i_{r-1}}\right) \operatorname{Lt}\left(f_{i_{r-1}}\right),
$$

that is

$$
\operatorname{Lt}\left(g_{i_{r}}\right) \in\left\langle\operatorname{Lt}\left(f_{i_{1}}\right), \ldots, \operatorname{Lt}\left(f_{i_{r-1}}\right)\right\rangle: \operatorname{Lt}\left(f_{i_{r}}\right)=\left\langle v_{i_{r} i_{1}}, \ldots, v_{i_{r} i_{r-1}}\right\rangle
$$

where for each $v_{k j}$, there exists $p_{k j}$ such that $v_{k j}$ and $p_{k j}$ are left useful for $\operatorname{Lm}\left(f_{k}\right)$ and $\operatorname{Lm}\left(f_{j}\right)$. We have seen in the proof of the left Buchberger's criterion that for each $j, \operatorname{Lm}\left(G_{k j}\right)=v_{k i} e_{k}$, according to the Lemma 2.13, there exists $j \in K \backslash\left\{i_{r}\right\}$ such that $L t\left(G_{i_{r} j}\right) \mid \operatorname{Lt}\left(g_{i_{r}}\right) e_{i_{r}}$ which contradict the fact that no term occurring in $G$ is divisible by any of the $L t\left(G_{k j}\right)$.

2) Assume that $\operatorname{Lm}\left(g_{i}\right) \operatorname{Lm}\left(f_{i}\right)=0 \forall i \in K$, then from (4) we have

$$
\text { (5) : } \sum_{i=i_{1}}^{i_{r}} g_{i}^{1} L t\left(f_{i}\right)+\sum_{i=i_{1}}^{i_{r}} \operatorname{Lt}\left(g_{i}\right) f_{i}=0
$$

where $g_{i}^{1}=\operatorname{tail}\left(g_{i}\right)$ and $\sum_{i=i_{1}}^{i_{r}} L t\left(g_{i}\right) \operatorname{tail}\left(f_{i}\right)=\sum_{i=i_{1}}^{i_{r}} L t\left(g_{i}\right) f_{i}=\sum_{i=i_{1}}^{i_{r}} L c\left(g_{i}\right) p_{i} S_{L}\left(f_{i}\right)$ where $\mathrm{p}_{\mathrm{i}}$ is a path exactly like in the proof of the left Buchberger's criterion. Since $\left\{\mathrm{f}_{1}, \ldots, \mathrm{f}_{\mathrm{s}}\right\}$ form a Groebner basis, by the left Buchberger's criterion we have $S_{L}\left(f_{i}\right)=\sum_{j=1}^{s} h_{j}^{i} f_{j}$ and $\operatorname{Lm}\left(S_{L}\left(f_{i}\right)\right)=\operatorname{Lm}\left(h_{k}^{i}\right) \operatorname{Lm}\left(f_{k}\right)$ for some k.

We transform (5) as follow $g_{i_{r}}^{1} L t\left(f_{r}\right)=\sum_{i=i_{1}}^{i_{r-1}} g_{i}^{1} L t\left(f_{i}\right)-\sum_{i=i_{1}}^{i_{r}} \sum_{j=1}^{S} L c\left(g_{i}\right) p_{i} h_{j}^{i} f_{j}$ therefore

$$
\operatorname{Lt}\left(g_{i_{r}}^{1}\right) \operatorname{Lt}\left(f_{r}\right) \in\left\langle\operatorname{Lt}\left(f_{1}\right), \ldots, \operatorname{Lt}\left(f_{s}\right)\right\rangle
$$

Using the Lemma 2.13 and the Proposition 2.5 we get a contradiction since no monomial occurring in the remainder is divisible by any of $\operatorname{Lm}\left(G_{i j}\right)$.

We give in the following example, an algorithm for computing a left Groebner basis for $\operatorname{syz}\left(f_{1}, \ldots, f_{S}\right)$.

Input: left Groebner basis $G=\left\{f_{1}, \ldots, f_{s}\right\}$ for $I=\langle G>$ and an admissible ordering $>$.

Output: left Groebner basis $T$ for $\operatorname{syz}\left(f_{1}, \ldots, f_{s}\right)$ w.r.t $>_{1}$ induced by $>$ and $G$.

$$
\begin{aligned}
& \text { (1) Set } T:=\emptyset:=H_{1}:=H_{2} \\
& \text { (2) For each } 1 \leq k \leq s \text {, do } \\
& \text { - }=S_{L}\left(f_{k}\right)=a_{k} f_{k}=\sum_{i=1}^{s} g_{i}^{k} f_{i} \\
& \text { - } r:=-g_{1}^{k} e_{1}-\cdots-\left(g_{k}^{k}-a_{k}\right) e_{k}-\cdots-g_{s}^{k} e_{s} \\
& \text { - } H_{1}:=H_{1} \cup\{r\} \text {. } \\
& \text { (3) For each } i=2, \ldots, s \text { and each minimal generator } v_{i j} \text { with } i>j \text {, do } \\
& \text { • }=S\left(f_{i}, f_{j}\right)=\frac{v_{j i}}{L c\left(f_{i}\right)} f_{i}-\frac{v_{i j}}{L c\left(f_{j}\right)} f_{j}=\sum_{i=1}^{s} p_{i}^{j i} f_{i} \\
& \text { - } s:=-p_{1}^{j i} e_{1}-\cdots-\left(p_{j}^{j i}+\frac{v_{i j}}{L c\left(f_{j}\right)}\right) e_{j}-\cdots-\left(p_{i}^{j i}-\frac{v_{j i}}{L c\left(f_{i}\right)}\right) e_{i}-\cdots-p_{s}^{j i} e_{s} ; \\
& \text { - } \mathrm{H}_{2}:=\mathrm{H}_{2} \cup\{s\} \\
& \text { (4) } T:=H_{1} \cup H_{2} \\
& \text { (5) Return } T \text {. }
\end{aligned}
$$

\section{Right Groebner Bases on $K Q$}


In this section we denote $I_{R}$ as a right ideal of $R=K Q$ and $>$ a right admissible ordering. We will omit some proofs and details in this section since they follow from the previous section.

Definition 3.1. Let $I_{R}$ be a right ideal of $R$ and $G$ be a subset of $I_{R}$. We say that $G$ is a right Groebner basis for $I_{R}$ with respect to a given right-admissible ordering if for every $f \in I_{R}$, there exists $g \in G$ such that $\operatorname{Lm}(g)$ divides $\operatorname{Lm}(f)$ i.e there exists $h \in R$ such that $\operatorname{Lm}(f)=\operatorname{Lm}(g) \operatorname{Lm}(h)$. In the other hand, $G$ is a right Groebner basis for $I_{R}$ if $\operatorname{Lm}\left(I_{R}\right)_{L}=\operatorname{Lm}(G)_{R}$.

Definition 3.2. Let $Q$ be a quiver. A cycle $c$ in $Q$ has an exit if there exists an edge in $Q$ not occurring in $c$ with its source in $c$. For example in the graph

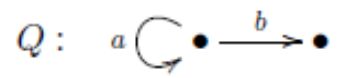

the cycle has an exit.

Proposition 3.3. The path $K$-algebra $K Q$ is right noetherian if and only if no cycle in $Q$ has an exit.

Proof. Similar with the left side.

In this section $R=K Q$ will be considered as right noetherian path $K$-algebra.

Proposition 3.4. Let $R$ be a right noetherian path $K$-algebra and < be a right admissible order.

1) A path $p \in T=<q_{i} / q_{i} \in \operatorname{path}(Q) \forall 1 \leq i \leq r>_{R}$ if and only if there exists $i_{0}$ such that $q_{i_{0}}$ divides $p$ on the right.

2) If $I_{R} \subset R$ is a right path ideal then $I_{R}$ has a unique finite minimal path generating set.

Proof. The proof is similar to the one of Proposition 2.5.

3.1. Right division's algorithm. Let $F=\left\{f_{1}, \ldots, f_{s}\right\}$ be a set of elements of $R=K Q$ and $>$ be a right admissible ordering.

Given element $g \in R \backslash\{0\}$, the following algorithm shows how to nd $q_{1}, \ldots, q_{s}, h \in K Q$ such that $g=f_{1} q_{1}+\cdots+$ $f_{s} q_{s}+h$ satisfying.

A1 if $h \neq 0$, then each term occurring in $h$ is not divisible by any of $\operatorname{Lt}\left(f_{i}\right) \forall 1 \leq i \leq s$.

A2 $\operatorname{Lm}(g) \geq \operatorname{Lm}\left(f_{i} q_{i}\right)$ for each $i \in\{1, \ldots, s\}$ since $q_{i}$ are obtained from $g$.

Input: $f, f_{1}, \ldots, f_{s} \in F$ and a right admissible ordering $>=\left(>_{1},>_{2}\right)$.

Output: $q_{1}, \ldots, q_{s}, h \in F$ such that $f=f_{1} q_{1}+\cdots+f_{s} q_{s}+h$.

Initialization: $q_{1}:=q_{2}:=\cdots:=q_{s}:=h:=0$ and $v:=f$;

- While $v \neq 0$ do

$-i:=1$;

- while $i \leq s$ do

* if $\operatorname{Lt}\left(f_{i}\right) \mid \operatorname{Lt}(v)$ then

$\cdot q_{i}:=q_{i}+\frac{\operatorname{Lt}(v)}{\operatorname{Lt}\left(f_{i}\right)}$

$\cdot v:=v-f_{i} \frac{\operatorname{Lt}(v)}{\operatorname{Lt}\left(f_{i}\right)}$

* Else

$\cdot i:=i+1$;

$-h:=h+\operatorname{Lt}(v)$

$-v:=v-\operatorname{Lt}(v)$

Proposition 3.5. Let $\left\{f_{1}, \ldots, f_{r}\right\} \subset R$ be a right Groebner basis for the ideal $I_{R}:=\left\langle f_{1}, \ldots, f_{r}\right\rangle_{L} \subset R$, $\rangle$ be a right admissible ordering and $g \in R \backslash\{0\} . g \in I_{L}$ if and only if the remainder $h$ of the division of $g$ by $f_{1}, \ldots, f_{r}$ is zero.

Definition 3.6. (Right useful paths)

Let $p, q \in \operatorname{path}(Q)$ be paths in $Q$. Two paths $u, v$ are called right useful for $p$ and $q$ with respect to the right admissible ordering <, if $u p=v q \neq 0$ and for any other paths $w_{1}$ and $w_{2}$ satisfying $p w_{1}=q w_{2} \neq 0$, we have $u$ divides $w_{1}$ and $v$ divides $w_{2}$ ( both on the right). 
Definition 3.7. (Right S-polynomial)

Let $f, g$ be non-zero polynomials and $<$ be a right admissible ordering. Let $u, v$ be right useful paths for $\operatorname{Lm}(f)$ and $\operatorname{Lm}(g)$ (i.e $\operatorname{Lm}(f) \cdot u=\operatorname{Lm}(g) \cdot v$ ), then the right S-polynomial $S_{R}(f, g)$ of $f$ and $g$ is dened as

$$
S_{R}(f, g)=f \cdot \frac{u}{L c(f)}-g \cdot \frac{v}{L c(g)} \text {. }
$$

Remark 3.8. If there exist no right useful paths for $\operatorname{Lm}(f)$ and $\operatorname{Lm}(g)$, we claim that $S_{R}(f, g)=0$.

Definition 3.9. (Right extended S-Polynomial)

Let $f \in R$ be a non-zero polynomial and $>$ be a right admissible ordering. We define the right extended S-polynomial $S_{R}(f)$ of $f$ as $S_{R}(f)=f \cdot p$ where $p$ is a path in $K Q$ such that $\operatorname{Lm}(f) \cdot p=0$ and $f \cdot p \neq 0$.

Remark 3.10. Let $p$ and $q$ be two paths satisfying the conditions of the Definition 3.9, if $q$ divides $p$ then we choose $S_{R}(f)=f$.

Definition 3.11. Let $I_{R}$ be a right path ideal and $w \in$ path $(Q)$. We define the left quotient ideal of $I_{R}$ and $w$ by $I_{R}: w=\left\{p \in \operatorname{path}(Q) / w \cdot p \in I_{R}\right\}$.

Lemma 3.12. Let $I_{R}=\left\langle w_{1}, \ldots, w_{r}\right\rangle_{L}$ be a right path ideal of $R, t$ be a monomial and $>$ be a right admissible ordering. Then the quotient ideal $I_{R}: t$ can be written as

$$
I_{R}: t=\left\langle v_{1}, \ldots, v_{l}\right\rangle_{L}
$$

where for each $1 \leq i \leq l \leq r$ there exists a monomial $p_{i}$ such that $t v_{i}=w_{i} p_{i}$ and $v_{i} p_{i}$ are right useful for $t$ and $w_{i}$.

Definition 3.13. Let $R=K Q$ and $T=R^{s}$ be a right free $R$-module with basis $\left\{e_{1}, \ldots, e_{s}\right\}$. By a monomial in $T$ involving the component $e_{j}$ we mean a component $e_{j}$ times a path in $R$.

Definition 3.14. (Right Syzygy)

Let $T=R^{s}$ be a right free $R$-module with basis $\left\{e_{1}, \ldots, e_{s}\right\}$ and let $I_{R}=\left\langle f_{1}, \ldots, f_{s}\right\rangle_{R}$ be a right ideal of $R$. By a right syzygy we mean an element of the kernel of the $R$-module homomorphism

$$
\begin{gathered}
\psi: T=R^{S} \rightarrow R \\
e_{i} \longmapsto f_{i} .
\end{gathered}
$$

We call $\operatorname{ker}(\psi)$ the (first) right syzygy module on $f_{1}, \ldots, f_{s}$ written $\operatorname{syz}\left(f_{1}, \ldots, f_{s}\right)_{R}=\operatorname{ker}(\psi)$.

Definition 3.15. (Induced ordering)

Let $G=\left\{f_{1}, \ldots, f_{s}\right\}$ be a set of polynomials and $>$ be a right admissible ordering. Let $T=R^{s}$ be a right free $R$-module with basis $\left\{e_{1}, \ldots, e_{s}\right\}$. We define the module ordering $>_{1}$ induced by $G$ and $>$ as follow: if $p, q$ are two paths in $R$ then

$$
\begin{gathered}
e_{i} p>_{1} e_{j} q \Leftrightarrow \operatorname{Lm}\left(f_{i}\right) p>p \operatorname{Lm}\left(f_{j}\right) p\left(\text { whenever } \operatorname{Lm}\left(f_{i}\right) p \neq 0 \neq \operatorname{Lm}\left(f_{j}\right)\right) q \text { or } \\
0 \neq \operatorname{Lm}\left(f_{i}\right) p=\operatorname{Lm}\left(f_{j}\right) q \text { and } i>j .
\end{gathered}
$$

Theorem 3.16. (Right Buchberger's criterion)

Let $f_{1}, \ldots, f_{s} \in R$ be non-zero polynomials and $>$ be a right admissible ordering on $R$. Then $\left\{f_{1}, \ldots, f_{s}\right\}$ form a right-Groebner basis for $I_{R}=\left\langle f_{1}, \ldots, f_{S}\right\rangle$ if and only if the following conditions are satisfied:

1) For each $i>j$, the remainder of $S_{R}\left(f_{i}, f_{j}\right)$ on right division's algorithm by $\left\{f_{1}, \ldots, f_{s}\right\}$ is zero.

2) For each $i$, the remainder of $S_{R}\left(f_{i}\right)$ on right division's algorithm by $\left\{f_{1}, \ldots, f_{s}\right\}$ is zero.

Input: give an ideal $I_{R}=\left\langle f_{1}, \ldots, f_{S}\right\rangle_{R} \subset R$ and a right admissible ordering $>=\left(>_{1},>_{2}\right)$ on $R$.

Output: a right Groebner basis for $I_{R}$.

Set $k=s$;

Set $P:=\left\{S_{R}\left(f_{i} f_{j}\right) / 1 \leq j<i \leq k\right\}$ and $Q:=\left\{S_{R}\left(f_{i}\right) / 1 \leq i \leq k\right\}$ 
A) Set $k=s$.

B) For $i=2, \ldots, k$, and for each minimal path generator $v_{j i}$ of

$$
M_{i}=\left\langle\operatorname{Lm}\left(f_{1}\right), \ldots, \operatorname{Lm}\left(f_{i-1}\right)\right\rangle_{R}: \operatorname{Lm}\left(f_{i}\right) \subset R,
$$

compute a remainder $h_{i j}$ as described in the Remark 2.18 above.

C) If some $h_{i j}$ is non-zero, set $k=k+1, f_{k}=h_{i j}$, and go back to B).

D) Return $\left\{f_{1}, \ldots, f_{k}\right\}$.

As in the previous section, we describe the right Schreyer's theorem

Theorem 3.17. (Schreyer's theorem)

If $\left\{f_{1}, \ldots, f_{s}\right\}$ is a right Groebner basis for $I_{R}=\left\langle f_{1}, \ldots, f_{s}\right\rangle_{R}$ w.r.t a right admissible ordering $>$, then the set $T=$ $\left\{G_{i j} H_{i} \in \operatorname{syz}_{L}\left(f_{1}, \ldots, f_{s}\right) / G_{i j} \neq 0 \neq H_{i}\right\}$ of right syzygies form a right Groebner basis for $\operatorname{syz}_{R}\left(f_{1}, \ldots, f_{s}\right)$ w.r.t the ordering $>_{1}$ induced by $>$ and $\left\{f_{1}, \ldots, f_{s}\right\}$.

\section{Computing Left and Right Syzygies}

In this section we propose a method for computing left and right syzygies of given polynomials $f_{1}, \ldots, f_{s} \in R$ $\backslash\{0\}$.

4.1. Left syzygies. In this subsection, we denote by $>$ a left admissible ordering and $f_{1}, \ldots, f_{s} \in R \backslash\{0\}$ polynomials. The goal of this subsection is to propose a method for computing left syzygies on $f_{1}, \ldots, f_{s}$.

Remark 4.1. Let $f_{1}, \ldots, f_{s} \in R \backslash\{0\}$ be polynomials and $G=\left\{f_{1}, \ldots, f_{s,} \ldots, f_{s^{\prime}}\right\}$ be a left Groebner basis for $\left\langle f_{1}, \ldots, f_{s}>_{L}\right.$ w.r.t $>$. Let $T=\left\{G_{i j}, H_{i} / G_{i j} H_{i} \in \operatorname{syz}(G)_{L} \backslash\{0\}\right\}$ be the set of left syzygies on the left Groebner bases $G$. Assume that we have $t$ such $G_{i j}, H_{i}$ arranged as follow:

By computing the left Groebner basis $G=\left\{f_{1}, \ldots, f_{s,}, \ldots, f_{s^{\prime}}\right\}$, we store each non-zero left syzygy $G_{i j}$ and $H_{i}$ such that those obtained from a left division leading to a new polynomial $f_{k}$ are fist and those obtained from a left division with remainder zero are second. The $G_{i j}$ fits as rows of the $t \times s^{\prime}$ matrix

$$
M_{t \times s^{\prime}}^{\prime}=\left(\begin{array}{ccccccccc}
g_{1}^{1} & g_{2}^{1} & \cdots & g_{s}^{1} & 1 & 0 & 0 & \cdots & 0 \\
g_{1}^{2} & g_{2}^{2} & \cdots & g_{s}^{2} & g_{s+1}^{2} & 1 & 0 & \cdots & 0 \\
\vdots & \vdots & \cdots & \vdots & \cdots & \vdots & & & \\
g_{1}^{s^{\prime}-s} & g_{2}^{s^{\prime}-s} & \cdots & g_{s}^{s^{\prime}-s} & g_{s+s}^{s^{\prime}-s} & g_{s+2}^{s^{\prime}-s} & g_{s+s}^{s^{\prime}-s} & \cdots & 1 \\
g_{1}^{s^{\prime}-s+1} & g_{2}^{s^{\prime}-s+1} & \cdots & g_{s}^{s^{\prime}-s+1} & g_{s+1}^{s^{\prime}-s+1} & g_{s+2}^{s^{\prime}-s+1} & g_{s+3}^{s^{\prime}+s+1} & \cdots & g_{s^{\prime}}^{s^{\prime}-s+1} \\
\vdots & \vdots & \cdots & \vdots & \cdots & \vdots & & & \\
g_{1}^{t} & g_{2}^{t} & \cdots & g_{s}^{t} & g_{s+1}^{t} & g_{s+2}^{t} & g_{s^{\prime}}^{t} & \cdots & g_{s^{\prime}}^{t}
\end{array}\right)
$$

Let

$$
\begin{aligned}
& A_{\left(s^{\prime}-s\right) \times s}^{\prime}=\left(\begin{array}{cccc}
g_{1}^{1} & g_{2}^{1} & \cdots & g_{s}^{1} \\
g_{1}^{2} & g_{2}^{2} & \cdots & g_{s}^{2} \\
\vdots & \vdots & \cdots & \vdots \\
g_{1}^{s^{\prime}-s} & g_{2}^{s^{\prime}-s} & \cdots & g_{s}^{s^{\prime}-s}
\end{array}\right), B_{\left(t-\left(s^{\prime}-s\right)\right) \times s}^{\prime}=\left(\begin{array}{cccc}
g_{1}^{s^{\prime}-s+1} & g_{2}^{s^{\prime}-s+1} & \cdots & g_{s}^{s^{\prime}-s+1} \\
\vdots & \vdots & \cdots & \vdots \\
g_{1}^{t} & g_{2}^{t} & \cdots & g_{s}^{t}
\end{array}\right),
\end{aligned}
$$

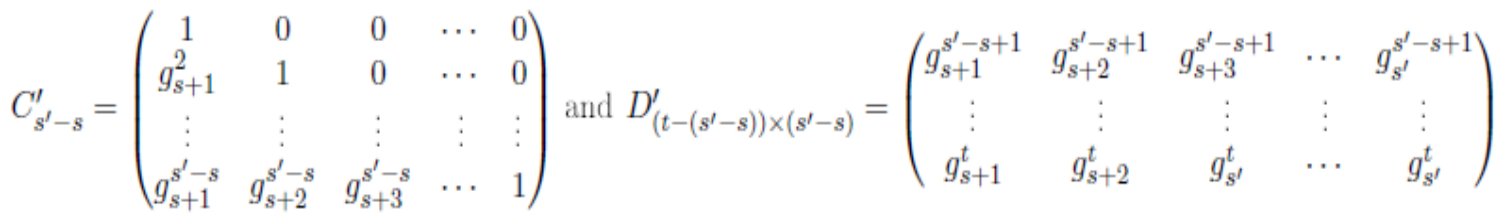

Then $M_{t \times s^{\prime}}^{\prime}$ can be regarded as the $t \times s^{\prime}$ block matrix

$$
M_{t \times s^{\prime}}^{\prime}=\left(\begin{array}{ll}
A^{\prime} & C^{\prime} \\
B^{\prime} & D^{\prime}
\end{array}\right)
$$


where $A^{\prime}=A_{\left(s^{\prime}-s\right) \times s}^{\prime}, B^{\prime}=B_{\left(t-\left(s^{\prime}-s\right)\right) \times s}^{\prime}, C^{\prime}=C_{s^{\prime}-s}^{\prime}$ and $D^{\prime}=D_{\left(t-\left(s^{\prime}-s\right)\right) \times\left(s^{\prime}-s\right)}^{\prime}$.

Theorem 4.2. With notations as above. Assume that the matrix $B^{\prime}$ is non-zero. The rows of the $\left(t-s^{\prime}+\right.$ $s) \times s$ matrix $B^{\prime}-D^{\prime} C^{\prime^{-1}} A^{\prime}$

form non-zero left syzygies on $f_{1}, \ldots, f_{s}$.

Proof. Set

$$
F=\left(\begin{array}{c}
f_{1} \\
f_{s}
\end{array}\right), F^{\prime}=\left(\begin{array}{l}
f_{s+1} \\
f_{s^{\prime}}
\end{array}\right) \text { and }\left(\begin{array}{c}
F \\
F^{\prime}
\end{array}\right)=\left(\begin{array}{c}
f_{1} \\
f_{s} \\
f_{s^{\prime}}
\end{array}\right) .
$$

We know by hypothesis that each row of the matrix

$$
\left(\begin{array}{ll}
A^{\prime} & C^{\prime} \\
B^{\prime} & D^{\prime}
\end{array}\right)
$$

form a left syzygy on $f_{1}, \ldots, f_{50}$. We can write

$$
\left(\begin{array}{ll}
A^{\prime} & C^{\prime} \\
B^{\prime} & D^{\prime}
\end{array}\right)\left(\begin{array}{l}
F \\
F^{\prime}
\end{array}\right)=\left(\begin{array}{l}
0 \\
0
\end{array}\right)
$$

then

$$
\begin{cases}A^{\prime} F+C^{\prime} F^{\prime}=0 \\ B^{\prime} F+D^{\prime} F^{\prime}=0\end{cases}
$$

Observe that $C^{\prime}$ is invertible, by multiplying each side of (1) by $C^{\prime^{-1}}$ on the left, we get $C^{\prime^{-1}} A F+F^{\prime}=$ $0 \Rightarrow F^{\prime}=-C^{\prime^{-1}} A F$ (3). Replacing (3) in (2) we get $\left(B^{\prime}-D^{\prime} C^{\prime^{-1}} A^{\prime}\right) F=0$ (4). finalement

Example 4.3. For the quiver $\Gamma$

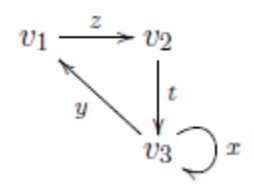

Let us compute a left Groebner basis for $F=\left\{f_{1}=z t+y z, f_{2}=t x+z, f_{3}=x^{2} y+t\right\}$ in $R=\mathbb{Q} \Gamma$ with respect to the left length lexicographic ordering $t>$ Ilex $Z>$ Ilex $Z>$ Ilex $y>$ llex $X>$ llex $V 1>$ llex $v 2>$ llex $v 3$.

Observe that $S_{L}\left(f_{2}\right)=v_{1} f_{2}=z=f_{4}, S_{L}\left(f_{3}\right)=v_{2} f_{3}=f_{5}$ and $S_{L}\left(f_{1}\right)=v_{3} f_{1}=y z=y f_{4}$. Observe that $S_{L}\left(f_{2}, f_{1}\right)=$

$S_{L}\left(f_{3}, f_{1}\right)=S_{L}\left(f_{3}, f_{2}\right)=S_{L}\left(f_{4}, f_{1}\right)=S_{L}\left(f_{4}, f_{2}\right)=S_{L}\left(f_{4}, f_{3}\right)=S_{L}\left(f_{5}, f_{2}\right)=S_{L}\left(f_{5}, f_{3}\right)=S_{L}\left(f_{5}, f_{4}\right)=0$ by the lack of useful paths. We have $S\left(f_{5}, f_{1}\right)=z f_{5}-v_{1} f_{1}=z t-z t=0$. The set $\left\{f_{1}, \ldots, f_{5}\right\}$ is then a left Groebner basis for the ideal $I_{L}=$ $\left\langle f_{1}, f_{2}, f_{3}>\right.$. From Schreyer's theorem, the set $\left\{v_{1} e_{2}-e_{4}, v_{2} e_{3}-e_{5}, v_{3} e_{1}-y e_{4},-v_{1} e_{1}+z e_{5}\right\}$ form a left Groebner basis for $\operatorname{syz}\left(f_{1}, \ldots, f_{5}\right)$ w.r.t the ordering induced by $>$ and $\mathrm{f} 1, \ldots, \mathrm{f} 5$.

Let us compute the set of syzygies for $f_{1}, f_{2} f_{3}$. We store each left syzygy of $f_{1}, \ldots, f_{5}$ as rows of the matrix

$$
M=\left(\begin{array}{ccccc}
0 & v_{1} & 0 & -1 & 0 \\
0 & 0 & v_{2} & 0 & -1 \\
v_{3} & 0 & 0 & -y & 0 \\
-v_{1} & 0 & 0 & 0 & z
\end{array}\right)
$$


Set

$$
A=\left(\begin{array}{ccc}
0 & v_{1} & 0 \\
0 & 0 & v_{2}
\end{array}\right), B=\left(\begin{array}{ccc}
v_{3} & 0 & 0 \\
-v_{1} & 0 & 0
\end{array}\right), C=\left(\begin{array}{cc}
-1 & 0 \\
0 & -1
\end{array}\right) \text { and } D=\left(\begin{array}{cc}
-y & 0 \\
0 & z
\end{array}\right)
$$

Observe that

$$
B-D C^{-1} A=\left(\begin{array}{ccc}
v_{3} & -y & 0 \\
-v_{1} & 0 & z
\end{array}\right)
$$

then $\operatorname{syz}\left(f_{1}, f_{2}, f_{3}\right)_{L}=<\left(v_{3},-y, 0\right),\left(-v_{1}, 0, z\right)>$.

4.2. Right syzygies. Let $R$ be a right noetherian path algebra and $f_{1}, \ldots, f_{s} \in R \backslash\{0\}$ be non-zero polynomials. The goal of this subsection is to propose a method for computing right syzygies on $f_{1}, \ldots, f_{s} \in R$.

Remark 4.4. Let $f_{1}, \ldots, f_{s} \in R \backslash\{0\}$ be non-zero polynomials and $G=\left\{f_{1}, \ldots, f_{s}, \ldots, f_{s^{\prime}}\right\}$ be a right Groebner basis for $\left\langle f_{1}, \ldots, f_{s}>_{R}\right.$ w.r.t the right admissible ordering <. Let $G_{i j}$ be right syzygies on $f_{1}, \ldots, f_{s}, \ldots, f_{s^{\prime}}$ for each pair $(i, j)$ $i>j$. We have seen in the right Schreyer's theorem that $\operatorname{syz}\left(f_{1}, \ldots, f_{S^{\prime}}\right)=\left\langle G_{i j} \neq 0 \neq H_{i} / \forall i>_{j}\right\rangle_{R}$. Assume that we have $t$ such $G_{i j} H_{i}$ arranged as follow:

By computing the right Groebner basis $G=\left\{f_{1}, \ldots, f_{s, \ldots,} f_{s^{\prime}}\right\}$, we store each non-zero right syzygy $G_{i j}$ and $H_{i}$ such that those obtained from a right division leading to a new polynomial $f_{k}$ are fist and those obtained from a right division with remainder zero are second. The $G_{i j}$ fits as columns of the $s^{\prime} \times t$ matrix

$$
M_{s^{\prime} \times t}=\left(\begin{array}{ccccccc}
g_{1}^{1} & g_{1}^{2} & \cdots & g_{1}^{s^{\prime}-s} & g_{1}^{s^{\prime}-s+1} & \cdots & g_{1}^{t} \\
g_{2}^{1} & g_{2}^{2} & \cdots & g_{2}^{s^{\prime}-s} & g_{2}^{s^{\prime}-s+1} & \cdots & g_{2}^{t} \\
\vdots & \vdots & \cdots & \vdots & \cdots & \vdots & \\
g_{s}^{1} & g_{s}^{2} & \cdots & g_{s}^{s^{\prime}-s} & g_{s}^{s^{\prime}-s+1} & \cdots & g_{s}^{t} \\
1 & g_{s+1}^{2} & \cdots & g_{s+1}^{s^{\prime}-s} & g_{s+1}^{s^{\prime}-s+1} & \cdots & g_{s+1}^{t} \\
0 & 1 & \cdots & g_{s+2}^{s^{\prime}-s} & g_{s+2}^{s^{\prime}-s+1} & \cdots & g_{s+2}^{t} \\
\vdots & 0 & \cdots & g_{s+3}^{s^{\prime}-s} & g_{s+3}^{s^{\prime}-s+1} & \cdots & g_{s+3}^{t} \\
\vdots & \vdots & \cdots & \vdots & \cdots & \vdots & \\
0 & 0 & \cdots & 1 & g_{s^{\prime}}^{s^{\prime}-s+1} & \cdots & g_{s^{\prime}}^{t}
\end{array}\right)
$$

Let

$$
\begin{aligned}
& A_{s \times\left(s^{\prime}-s\right)}=\left(\begin{array}{cccc}
g_{1}^{1} & g_{1}^{2} & \cdots & g_{1}^{s^{\prime}-s} \\
g_{2}^{1} & g_{2}^{2} & \cdots & g_{2}^{s^{\prime}-s} \\
& & \cdots & \\
g_{s}^{1} & g_{s}^{2} & \cdots & g_{s}^{s^{\prime}-s}
\end{array}\right), B_{s \times\left(t-\left(s^{\prime}-s\right)\right)}=\left(\begin{array}{lll}
g_{1}^{s^{\prime}-s+1} & \cdots & g_{1}^{t} \\
g_{2}^{s^{\prime}-s+1} & \cdots & g_{2}^{t} \\
& \cdots & \\
g_{s}^{s^{\prime}-s+1} & \cdots & g_{s}^{t}
\end{array}\right), \\
& D_{\left(s^{\prime}-s\right) \times\left(t-\left(s^{\prime}-s\right)\right)}=\left(\begin{array}{lll}
g_{s+1}^{s^{\prime}-s+1} & \cdots & g_{s+1}^{t} \\
g_{s+2}^{s^{\prime}-s \text { and }} & \cdots & g_{s+2}^{t} \\
& \cdots & \\
g_{s^{\prime}}^{s^{\prime}-s+1} & \cdots & g_{s^{\prime}}^{t}
\end{array}\right)
\end{aligned}
$$




$$
C_{s^{\prime}-s}=\left(\begin{array}{cccc}
1 & g_{s+1}^{2} & \cdots & g_{s+s}^{s^{\prime}-s} \\
0 & 1 & \cdots & g_{s+2}^{s^{\prime}-s} \\
\vdots & 0 & \cdots & g_{s+3}^{s^{\prime}-s} \\
\vdots & \vdots & \cdots & \vdots \\
0 & 0 & \cdots & 1
\end{array}\right)
$$

Then $M_{s^{\prime} \times t}$ can be regarded as the $s^{\prime} \times t$ block matrix

$$
M_{s^{\prime} \times t}=\left(\begin{array}{cc}
A_{s \times\left(s^{\prime}-s\right)} & B_{s \times\left(t-\left(s^{\prime}-s\right)\right)} \\
C_{s^{\prime}-s} & D_{\left(s^{\prime}-s\right) \times\left(t-\left(s^{\prime}-s\right)\right)}
\end{array}\right) .
$$

Notation 4.5. For simplicity, we denote $\mathrm{A}=A_{s \times\left(s^{\prime}-s\right)}, \mathrm{B}=B_{s \times\left(t-\left(s^{\prime}-s\right)\right)}, \mathrm{C}=C_{s^{\prime}-s}$ and $\mathrm{D}=D_{\left(s^{\prime}-s\right) \times\left(t-\left(s^{\prime}-s\right)\right)}$.

Theorem 4.6. With notations as above. The columns of the $s \times\left(t-s^{\prime}+s\right)$ matrix $B-A C^{-1} D$ are right syzygies on $f_{1}, \ldots, f_{s}$.

Proof. Set

$$
\begin{aligned}
& F=\left(f_{1}, \ldots, f_{s}\right), F^{\prime}=\left(f_{s+1}, \ldots, f_{s^{\prime}} \text { and }\left(F \quad F^{\prime}\right)=\right. \\
& \left(f_{1} \ldots f_{s} \ldots f_{s^{\prime}}\right) .
\end{aligned}
$$

We know by hypothesis that each column of the matrix

$$
\left(\begin{array}{ll}
A & B \\
C & D
\end{array}\right)
$$

form a right syzygy on $f_{1}, \ldots, f_{s^{\prime}}$. We can write

$$
\left(\begin{array}{ll}
F & F^{\prime}
\end{array}\right)\left(\begin{array}{ll}
A & B \\
C & D
\end{array}\right)=\left(\begin{array}{ll}
0_{1 \times\left(s^{\prime}-s\right)} & 0_{1 \times\left(t-s^{\prime}+s\right)}
\end{array}\right)
$$

then

$$
\left\{\begin{array}{l}
F A+F^{\prime} C=0_{1 \times\left(s^{\prime}-s\right)} \\
F B+F^{\prime} D=0_{1 \times\left(t-s^{\prime}+s\right)}
\end{array}\right.
$$

Note that $C$ is invertible, by multiplying each side of (1) by $C^{-1}$ on the right, we get $F A C^{-1}+F^{\prime}=0_{1 \times\left(s^{\prime}-s\right)}$ (3) . By multiplying each side of (3) on the right by $D$, we get $F A C^{-1} D+F^{\prime} D=0_{1 \times\left(t-s^{\prime}+s\right)}$ (4). Subtracting (2) and (4) side by side we get $F B-F A C^{-1} D=F\left(B-A C^{-1} D\right)=0_{1 \times\left(t-s^{0+s}\right)} \quad$ (6). Thus, each column of the matrix $B$ - $A C^{-1} D$ is a right syzygy on $f_{1}, \ldots, f_{s}$.

Example 4.7. For the quiver $\Gamma$

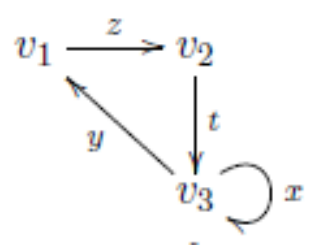

Let us compute a right Groebner basis for $F=\left\{f_{1}=z t+y z, f_{2}=t x+z, f_{3}=x^{2} y+t\right\}$ in $R=\mathbb{Q} \Gamma$ with respect to 
the right length lexicographic ordering $t>\operatorname{rlex} Z>\operatorname{rlex} Z>\operatorname{rlex} y>$ rlex $X>$ rlex $v_{1}>$ rlex $v_{2}>$ rlex $v_{3}$.

Observe that $S_{R}\left(f_{1}\right)=f_{2} v_{2}=y z=f_{4}, S_{R}\left(f_{2}\right)=f_{2} v_{2}=z=f_{5}$ and $S_{R}\left(f_{3}\right)=f_{3} v_{3}=t=f_{6}$.

By the lack of right useful paths, only the following right $S$-polynomials are computable:

$S_{R}\left(f_{5}, f_{1}\right)=f_{5} t-f_{1} v_{3}=0$ and $S_{R}\left(f_{6}, f_{2}\right)=f_{6} x-f_{2} v_{3}=0$, for the others, we claim them to be zero. The set $\left\{f_{1}, \ldots, f_{6}\right\}$ is then a right Groebner basis for $\left\langle f_{1}, f_{2}, f_{3}>_{R}\right.$.

Let us compute the set of syzygies for $f_{1}, f_{2}, f_{3}$. We store each right syzygy of $f_{1}, \ldots, f_{6}$ as column of the matrix

$$
M=\left(\begin{array}{ccccc}
v_{2} & 0 & 0 & -v_{3} & 0 \\
0 & v_{2} & 0 & 0 & -v_{3} \\
0 & 0 & v_{3} & 0 & 0 \\
-1 & 0 & 0 & 0 & 0 \\
0 & -1 & 0 & t & 0 \\
0 & 0 & -1 & 0 & x
\end{array}\right) .
$$

Set

$$
A=\left(\begin{array}{ccc}
v_{2} & 0 & 0 \\
0 & v_{2} & 0 \\
0 & 0 & v_{3}
\end{array}\right), B=\left(\begin{array}{cc}
-v_{3} & 0 \\
0 & -v_{3} \\
0 & 0
\end{array}\right), C=\left(\begin{array}{ccc}
-1 & 0 & 0 \\
0 & -1 & 0 \\
0 & 0 & -1
\end{array}\right) \text { and } D=\left(\begin{array}{cc}
0 & 0 \\
t & 0 \\
0 & x
\end{array}\right) \text {. }
$$

Observe that

$$
B-A C^{-1} D=\left(\begin{array}{cc}
-v_{3} & 0 \\
t & -v_{3} \\
0 & x
\end{array}\right)
$$

then $\operatorname{syz}\left(f_{1}, f_{2}, f_{3}\right)_{R}=<\left(-v_{3}, t, 0\right),\left(0,-v_{3}, x\right)>$.

\section{Conlusion}

This paper propose an algorithm for computing the left (resp. right) syzygies modules in a path $K$-algebra $K Q$, this gives necessary tools for working on an algorithm for computing a generating set of the intersection of left (resp. right) ideals in $K Q$. This paper also provides ideas that can be used to think of an algorithm for computing the two-sided syzygies modules. Since for any quiver $Q$ we can define the associated Leavitt path $K$-algebra $L_{K}(Q)$ which is nothing but a path $K$-algebra on the extending quiver of $Q$, and satisfy the Cunz-Krieger relations, this paper can be the first step for studying the theory of Groebner bases and applications in $L_{K}(Q)$.

\section{Acknowledgment}

Authors are grateful to the African Institute for Mathematical Sciences for giving to the first author the opportunity to visit the second author at the AIMS research centre during the preparation of this paper.

\section{References}

[1] Farkas, D., Feustel, C., \& Green, E. L. (1993). Synergy in the theories of Groebner bases and path algebras. Can. J. Math, 45, 727-739.

[2] Green, E. (2000). Multiplication bases, Groebner bases, and right Groebner bases. Journal of Symbolic computation, 29, 601-623.

[3] Green, E. L. (1999). Non-commutative Groebner bases and projective resolutions. Computaional Methods for Representations of Groups and Algebras, Progress in Math, 173, 29-60. 
[4] Leamer, M. J. (2006). Groebner nite path algebras. Journal of Symbolic Computation, 41, 98-111.

[5] Levandovsky, V. (2005). Non-commutative computer algebra for polynomial algebras: Groebner bases, applications and implementation. Doctoral thesis, Fachbereich Mathematik, Technische Universitaet Kaiserslautern.

[6] Li, H. (2011). Groebner bases in ring theory. World Scientic Publishing Co Pte Ltd, 57-120.

[7] Mialebama, B. A. S. E., \& Sow, D. (2014). Noncommutative Groebner bases over rings. Communications in Algebra, 43(2), 541-557.

[8] Bluhm, H. (2005). Syzygienberechnung ueber nicht-kommutativen polynomringen. Diploma thesis, Fachbereich Mathematik, Universitaet Dortmund.

[9] Decker, W., \& Schreyer, F. 0. (2015). Varieties, Groebner bases, and algebraic curves. Book in Preparation.

[10] Eisenburd, D. (2005). The geometry of syzygies: a second course in algebraic geometry and commutative algebra. Graduate Texts in Mathematics, 229.

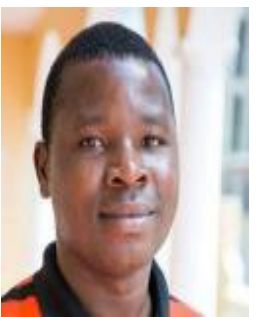

Sylvain Attan was born in Djidja (Rep. of Benin) on November, $15^{\text {th }}$ 1987. He is a $\mathrm{PhD}$ holder in mathematics (non-associative algebra), obtained at the Institute of Mathematics and Physical Sciences of the University of Abomey-Calavi (Benin), in March 2015. After his defence, he visited twice the African Institute for Mathematical Sciences in South Africa during the preparation of this paper. He is currently a lecturer and researcher at the University of Abomey-Calavi (Benin ).

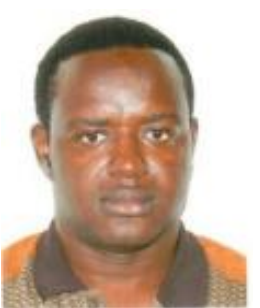

Andre Saint Eudes Mialebama Bouesso was born in Brazzaville (Rep. of Congo) on November, 2nd 1981. He is a PhD holder in mathematics (computer algebra), obtained in Cheikh Anta Diop University from Dakar (Senegal) in March 2014. After his defence, he was a DAAD postdoc research fellow at the Institute of Mathematics and Physical Sciences from Porto Novo (Benin) from June to November 2014. He is currently a postdoc research fellow at the African Institute for Mathematical Sciences in South Africa from January 2015 to December 2017. From January 2018, he will be a permanent researcher and lecturer in Marien Ngouabi University from Brazzaville (Rep. of Congo). 